\title{
Cavernous Angioma with Olivary Hypertrophy and Contralateral Cerebellar Diaschisis
}

\author{
Yuichi Komaba, Tatsuya Nomoto, Shin Kitamura and Akiro Terashi
}

\begin{abstract}
We describe a 66-year-old man with a 20-year history of ataxic gait who suddenly developed diplopia on rightward gaze. Neurologic examination revealed right hemi-ataxia and hemihypesthesia, and left internuclear ophthalmoplegia. MRI showed a cavernous angioma in the left tectum, mild right cerebellar atrophy, and left interior olivary hypertrophy. Single photon emission computed tomography (SPECT) imaging demonstrated contralateral cerebellar diaschisis. We discuss the findings and review the literature concerning contralateral cerebellar diaschisis.
\end{abstract}

(Internal Medicine 36: 504-507, 1997)

Key words: single photon emission computed tomography (SPECT), dentato-olivary pathway

\section{Introduction}

Crossed cerebellar diaschisis is defined as functional impairment in the cerebellum, which is remote from the causative lesion; it is often demonstrated in patients with contralateral supratentorial lesions on single photon emission computed tomography (SPECT) or positron emission tomography (PET) imaging (1). Involvement of the cerebropontine-cerebellar tract is thought to result in crossed cerebellar diaschisis; however, infratentorial lesions interrupting the cerebropontine-cerebellar tract may also result in contralateral cerebellar diaschisis in some patients (2-4) [It may be more precise to refer to cerebellar diaschisis with contralateral supratentorial lesion as "crossed cerebellar diaschisis", and cerebellar diaschisis with contralateral infratentorial lesion as "contralateral cerebellar diaschisis"]. Here, we describe a patient with a tegmental lesion who appears to have developed contralateral cerebellar diaschisis as a result of interruption of the dentato-olivary pathway.

\section{Case Report}

A 66-year-old man with a 20-year history of ataxic gait, presented with sudden onset of diplopia on rightward gaze. He was admitted to the Nippon Medical School Hospital 2 days after onset of symptoms. No history of specific disease except for right tympanitis at the age of 62 . His father died from stroke at the age of 56, his mother died from cancer at the age of 86 , and his siblings are healthy.
His blood pressure was $160 / 88 \mathrm{mmHg}$, and his pulse rate was 90/min and regular. Physical examination revealed no abnormal findings in his chest and abdomen. On neurologic examination, no pathologic reflexes were elicited. Muscle tone, deep tendon reflexes, and muscle strength were normal in all extremities. There was right hemi-ataxia and right hemihypesthesia to vibration and touch. Movement of the right upper extremity was accompanied by an intention tremor, and his gait was ataxic with rightward deviation. No palatal myoclonus was present. Although left internuclear ophthalmoplegia was found, the ocular position was normal.

Tests of cranial nerves revealed impaired hearing on the right due to tympanitis. Except for zinc sulfate turbidity test (ZTT), $\gamma$-glutamyl transpeptidase $(\gamma$-GTP), and blood urea nitrogen (BUN), the laboratory findings including blood count, urinalysis and blood biochemical profile were normal on admission (ZTT=12.1 U, $\gamma-\mathrm{GTP}=47 \mathrm{IU} / \mathrm{l}, \mathrm{BUN}=22.3 \mathrm{mg} / \mathrm{dl}$ ). Magnetic resonance imaging (MRI) was performed 3 weeks after the onset using a $1.0 \mathrm{~T}$ (Impulse Extra, Siemens Co.) system. On $\mathrm{T}_{2}$-weighted MR images, there was a heterogeneous high signal intensity lesion surrounded by a low intensity rim in the left tectum (Fig. 1A, B). There was also hypertrophy of the left inferior olive (Fig. 1C) and mild right cerebellar atrophy (Fig. 1D). On $\mathrm{T}_{2}$-weighted MR images, the left inferior olivary nucleus showed high signal intensity (Fig. 1E). Regional cerebral blood flow ( $\mathrm{rCBF}$ ) was measured utilizing the method described by Iida et al (5). For data acquisition, a SPECT scan was performed after intravenous injection of $\mathrm{N}$ -

From the Second Department of Internal Medicine, Nippon Medical School, Tokyo

Received for publication November 5, 1996; Accepted for publication April 3, 1997

Reprint requests should be addressed to Dr. Yuichi Komaba, the Second Department of Internal Medicine, Nippon Medical School, 3-3-5 Iidabashi, Chiyodaku, Tokyo 102 

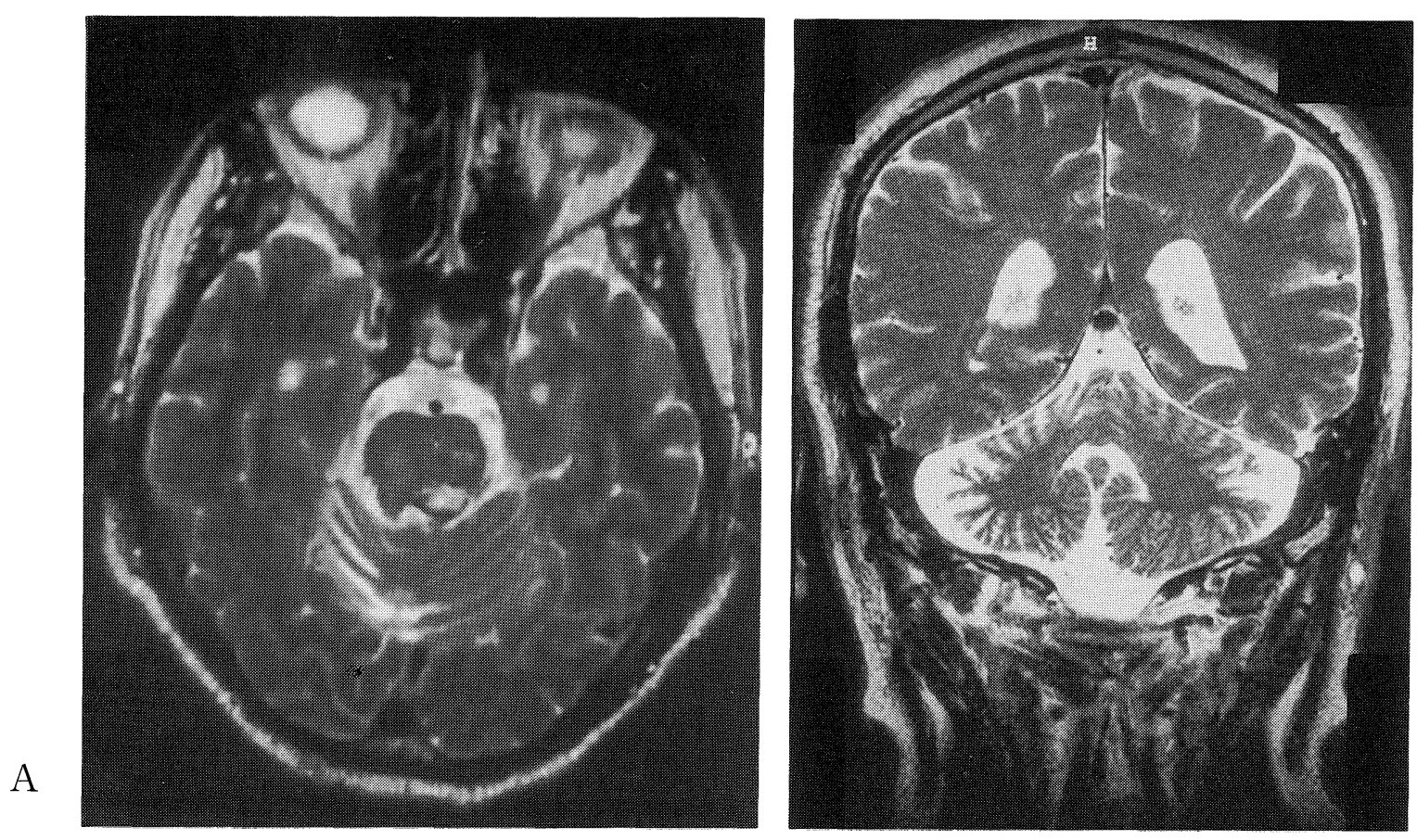

$\mathrm{D}$
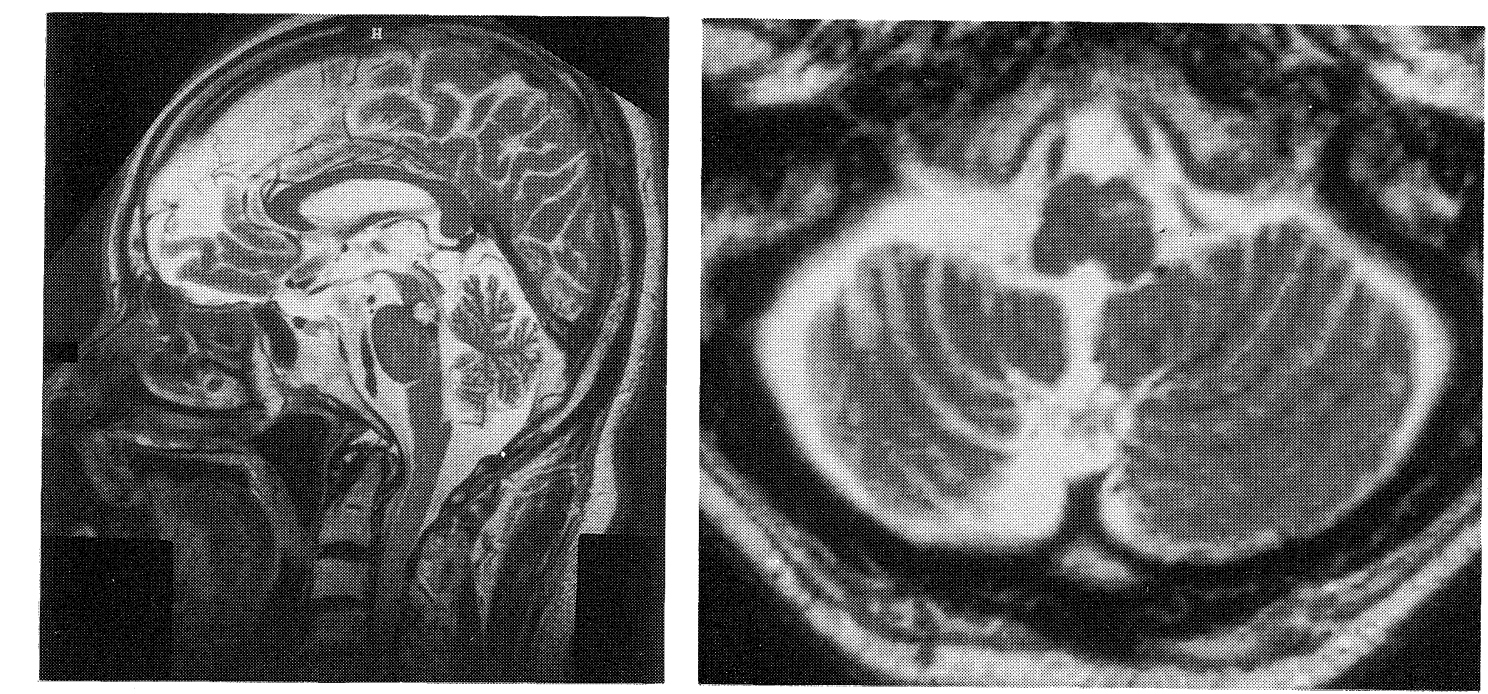

$\mathrm{E}$

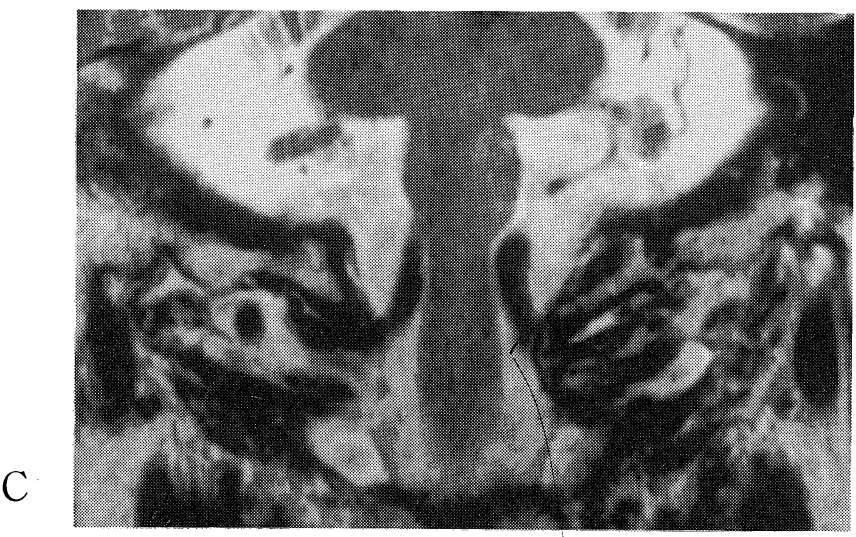

Figure 1. A 66-year-old man with right hemi-ataxia and hemi-hypesthesia, and left internuclear ophthalmoplegia. (A) MR image at the level of the upper pons ( $T_{2}$-weighted, axial, $T R: 3,800$ $\mathrm{ms}$, TE: $120 \mathrm{~ms}$ ) shows a heterogeneous high signal intensity lesion in the left tegmentum surrounded by a low intensity rim. (B) Sagittal MR image ( $T_{2}$-weighted, $T R: 3,800 \mathrm{~ms}$, TE: $120 \mathrm{~ms}$ ) shows the lesion located in the left side of the tegmentum, and there was no evidence of invasion into the pontine nucleus. (C) Coronal MR image ( $\mathrm{T}_{2}$-weighted, TR: 3,900 ms, TE: $120 \mathrm{~ms}$ ) shows obvious hypertrophy of the left inferior olive. (D) Coronal MR image $\left(\mathrm{T}_{\mathbf{2}^{-}}\right.$ weighted, TR: 3,900 ms, TE: $120 \mathrm{~ms}$ ) shows mild right cerebellar atrophy. (E) MR image at the level of the medulla oblongata $\left(\mathbf{T}_{2^{-}}\right.$ weighted, axial, TR: $3,800 \mathrm{~ms}$, TE: $120 \mathrm{~ms}$ ) shows hypertrophy of the left inferior olive as a high signal intensity area. 


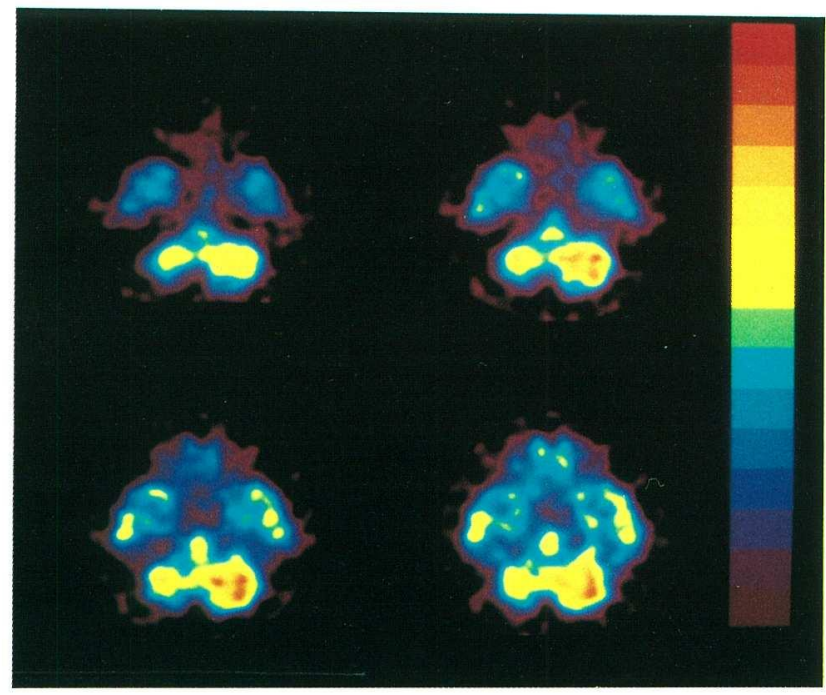

Figure 2. IMP autoradiographic SPECT study demonstrates a reduction of $\mathrm{CBF}$ in the right cerebellum $(\mathrm{R} / \mathrm{L}$ of cerebellum $=$ 0.81) ( $\mathrm{rCBF}$ image).

isopropyl-p- $\left[{ }^{123} \mathrm{I}\right]$ iodoamphetamine $\left({ }^{123} \mathrm{I}\right.$-IMP). The SPECT scanner was a Headtome-SET080 (Simadzu Co.). We injected $222 \mathrm{MBq}$ of ${ }^{123} \mathrm{I}$-IMP intravenously as a tracer and measured rCBF (pitch $10 \mathrm{~mm}$, 31 slices, parallel with $\mathrm{OM}$ line). We measured the radioactivity of arterial blood sampled from the left brachial artery 10 minutes after the start of injection, using a scintillator. We started to acquire images 25 minutes after the start of injection and continued to acquire images for 30 minutes. CBF images were created with the SPECT autoradiographic method (5). A circular region of interest (ROI) of $22 \mathrm{~mm}$ diameter was placed on each cerebellar hemisphere in the $\mathrm{CBF}$ images and each $\mathrm{rCBF}$ was calculated. The $\mathrm{rCBF}$ of the right cerebellar hemisphere was $37.9 \mathrm{ml} / 100$ $\mathrm{g} / \mathrm{min}$ and that of the left hemisphere was $47.2 \mathrm{ml} / 100 \mathrm{~g} / \mathrm{min}$ (left to right ratio, 0.81). SPECT images (Fig. 2) also showed a reduction of $\mathrm{CBF}$ in the right cerebellar hemisphere confirming the contralateral cerebellar diaschisis.

\section{Discussion}

MR images of this patient revealed the left tegmental lesion that showed heterogeneous high signal intensity on $\mathrm{T}_{2}$-weighted images, surrounded by a low signal intensity rim. The lesion had the typical appearance of a cavernous angioma. The patient's 20-year history of ataxia supports the view that this lesion was longstanding; however, the sudden onset of internuclear ophthalmoplegia suggests recent bleeding or ischemia. However, because either $\mathrm{T}_{1}$-weighted or $\mathrm{T}_{2}$-weighted images of a patient with the subacute stage of cerebral hemorrhage show a homogenous high signal intensity which is a reflection of a pooling of dilute-free methemoglobin, the lesion with a heterogenous high signal intensity may not suggest bleeding.

Hypertrophic olivary degeneration is considered to be trans- synaptic degeneration of the dentato-olivary pathway (8). The dentato-olivary pathway extends from the dentate nucleus to the contralateral inferior olive, coursing in the superior cerebellar peduncle, crossing the midline at its decussation (the commissure of Wernekink), passing by the internal and dorsal surface of the red nucleus, and becoming part of the central tegmental tract of the contralateral side. Olivary hypertrophy can be seen with lesions of the dentate nucleus or central tegmental tract and is characterized by enlargement of neurons with vacuolation and bizarre shapes, and enlargement of astrocytes with bizarre nuclei and prominent thick processes (7). Astrocytes are increased in number and multinucleated, and often there is severe fibrillary gliosis (8-10). The high signal intensity in the hypertrophic olivary nuclei on $\mathrm{T}_{2}$-weighted and proton density-weighted images appears to be due to gliosis and increased intracellular water content (11-14). In the present patient, the coronal MR images demonstrated obvious hypertrophy of the inferior olivary nucleus opposite to the side of the lesion of the central tegmental tract, and axial $\mathrm{T}_{2}$-weighted images demonstrated the hypertrophic olive as an area of high signal intensity. Gautier and Blackwood described patients with lesions in the central tegmental tract who had ipsilateral olivary hypertrophy and pallor of myelin staining surrounding the contralateral dentate nucleus (15). Kim et al (10) showed that in patients with lesions of the central tegmental tract the relative hypointensity of the contralateral dentate nucleus on $\mathrm{T}_{2}$-weighted images was due to damage of axonal transport that caused reduction of iron accumulation in the dentate nucleus.

Interruption of the cerebropontine-cerebellar tract is thought to be the most likely mechanism for crossed cerebellar diaschisis. SPECT or PET imaging often demonstrates crossed cerebellar diaschisis in patients with contralateral cerebrocortical lesions. The cerebropontine-cerebellar tract derives from extensive areas of the cerebrum, passes through the medial and lateral aspect of the cerebral peduncle, and projects to the ipsilateral pontine nuclei located ventral to the medial lemniscus (6). Pontine nuclei send axons to the contralateral cerebellum via the middle cerebral peduncle, traversing the anterior pons as the transverse pontine fibers. This explains how either a supratentorial lesion may cause crossed cerebellar diaschisis or an infratentorial lesion may cause contralateral cerebellar diaschisis. In patients with a unilateral pontine lesion, contralateral cerebellar diaschisis and ipsilateral supratentorial hypoperfusion have been observed $(3,6)$. The lesions of the cerebropontine-cerebellar tract within the pons are thought to produce contralateral cerebellar diaschisis. In addition, lesions in the thalamus also cause crossed cerebellar diaschisis, and this has been attributed to disruption of the dentato-rubro-thalamic pathway (2). In the present patient, MRI demonstrated that the lesion was located in the left central tegmental tract and invaded the left medial lemniscus. Since the lesion did not involve the pontine nucleus, this patient's CCD could not be due to interruption of the cerebropontine-cerebellar tract. Though there may be no fibers of the dentate-olivary pathway which precisely correspond to fibers of the dentato-rubro-thalamic pathway, the course from the dentate nucleus to the red nucleus is common. 


\section{Cavernous Angioma with Olivary Hypertrophy and Diaschisis}

Therefore, we postulate that our patient's contralateral cerebellar diaschisis may have resulted from interruption of the dentatoolivary pathway, similar to interruption of the dentato-rubrothalamic pathway.

\section{References}

1) Baron JC, Bousser MG, Comar D, Duquesnoy N, Sastre J, Castaigne P. "Crossed cerebellar diaschisis": A remote functional suppression secondary to supratentorial infarction in man. J Cereb Blood Flow Metab 1: s500, 1981.

2) Tanaka $M$, Kondo $S$, Hirai $S$, Ishiguro $K$, Ishihara $T$, Morimatsu $M$. Crossed cerebellar diaschisis accompanied by hemiataxia: A PET study. J Neurol Neurosurg Psychiatry 55: 121, 1992.

3) Fazekas F, Payer F, Valetitsch H, Schmidt R, Flooh E. Brain stem infarction and diaschisis: A SPECT cerebral perfusion study. Stroke 24: 1162, 1993.

4) Perani D, Lucignani G, Pantano P, Gerundini P, Lenzi GL, Fazio F. Cerebellar diaschisis in pontine ischemia: A case report with singlephoton emission computerized tomography. J Cereb Blood Flow Metab 7: 127, 1987.

5) Iida $\mathrm{H}$, Itoh $\mathrm{H}$, Nakazawa $\mathrm{M}$, et al. Quantitative mapping of regional cerebral blood flow using iodine-123-IMP and SPECT. J Nucl Med 35: 2019, 1994.
6) Brodal A. Cerebrocerebellar pathways: Anatomical data and some functional implications. Acta Neurol Scan Suppl 51: 153, 1972.

7) Sohn D, Levine S. The hypertrophy of the olive. A report of 43 cases. in: Zimmermann HM (ed) Progress in Neuropathology, vol 3. Grune and Stratton, New York, 202, 1971.

8) Lapresle J, Hamida MB. The dentato-olivary pathway: Somatotopic relationship between the dentate nucleus and the contralateral inferior olive. Arch Neurol 22: 135, 1970.

9) Kitajima M, Korogi $Y$, Shimomura O, et al. Hypertrophic olivary degeneration: MR imaging and pathologic findings. Radiology 192: 539, 1994.

10) Kim SJ, Lee JH, Suh DC. Cerebellar MR changes in patients with olivary hypertrophic degeneration. AJNR Am J Neuroradiol 15: 1715, 1994.

11) Revel MP, Mann M, Brugieres P, Poirier J, Gaston A. MR appearance of hypertrophic olivary degeneration after contralateral cerebellar hemorrhage. AJNR Am J Neuroradiol 12: 71, 1991.

12) Uchino $A$, Hasuo $K$, Uchida $K$, et al. Olivary degeneration after cerebellar or brain stem hemorrhage: MRI. Neuroradiology 35: 335, 1993.

13) Yokota T, Hirashima F, Fukukawa T, Tsukagoshi H, Yoshikawa H. MRI findings of inferior olives in palatal myoclonus. J Neurol 236: 115, 1989.

14) Pierot L, Cervera-Pierot P, Duyckaerts C, Chiras J, Brunet P. Palatal myoclonus and inferior olivary lesions: MRI-pathologic correlation. J Comput Assist Tomogr 16: 160, 1992.

15) Gautier JC, Blackwood W. Enlargement of the inferior olivary nucleus in association with lesions of the central tegmental tract or dentate nucleus. Brain 84: 23, 1961. 